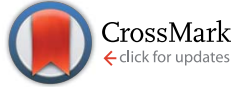

Cite this: RSC Adv., 2017, 7, 17417

\title{
New carbon allotropes with metallic conducting properties: a first-principles prediction $\uparrow$
}

\author{
Xinyu Fan, Jiao Li and Gang Chen*
}

Combining density functional theory computation and the global minimum structural search, we have found new $\alpha^{-}, \beta-$, and $\delta$-phographene carbon allotropes. The $\alpha$-phographene is almost degenerate in energy with the previously reported phagraphene, which is energetically favorable as compared to the $\beta$ and $\delta$-phographenes and the other known non-honeycomb two-dimensional carbon allotropes. Our careful studies of the $\alpha$ - and $\beta$-phographenes show good dynamic, thermal, and mechanical stabilities. The pristine $\alpha$ - and $\beta$-phographenes are metallic, which would be altered to wide-gap semiconductors by hydrogenation. Additionally, the $\alpha$-phographene shows enhanced joint density of states at the energy of $\sim 1.6 \mathrm{eV}$, which may find applications in photoelectronics. The analyses on the growth of structural motifs and the effects of net charge suggest the possibilities for experimentally fabricating $\alpha$ phographene. Interestingly, the $\alpha$-phographene has a Young's modulus and Poisson's ratio close to those of graphene, showing remarkable mechanical properties. It may also find applications in supporting $\mathrm{Au}$ or Pt clusters as high-performance complex catalyst nanostructures, calling for further investigations on both theory and experiment.

Received 12th February 2017 Accepted 10th March 2017

DOI: 10.1039/c7ra01762f

rsc.li/rsc-advances had been extensively studied in both theory and experiment, the first two-dimensional carbon sheet was only successfully synthesized on experiment in 2004 by Novoselov et al. ${ }^{4}$ Preserved by both inversion symmetry and time reversal symmetry, the conjugate $\pi$ and $\pi^{*}$ bands touch at Fermi energy to form Dirac cones at $K$ and $K^{\prime}$ points in reciprocal space with nearly linear dispersion, resulting in high mobility massless charge carriers. The success of graphene has motivated extensive researches for newer forms of $2 \mathrm{D}$ materials.

The outstanding progress in the research field of graphene has also inspired the investigations of various $2 \mathrm{D}$ carbon allotropes. Besides the hexagonal graphene with ideal $\mathrm{sp}^{2}$ electronic configuration, the experimentalist has the methodologies to generate networks of combinations of $\mathrm{sp}, \mathrm{sp}^{2}$ and $\mathrm{sp}^{3}$ hybridized carbons. ${ }^{5}$ Carbon that can form a variety of polygons ranging from linear chains to dodecagons attracts a lot of attentions in exploring new carbon planar nanostructures, which are, for example, the graphyne, ${ }^{10-20}$ graphdiyne, ${ }^{20-23}$ graphenylene ${ }^{24,25}$ biphenylene, ${ }^{26,27}$ radialenes,${ }^{28}$ pentagraphene, ${ }^{29}$ pentahexoctite, ${ }^{30}$ HOP-graphene, ${ }^{31}$ pentaheptities, ${ }^{32,33}$ haeckelites, ${ }^{34}$ squarographenes, ${ }^{35}$ planar $\mathrm{C}_{4}$ sheet, ${ }^{36-42}$ OP-graphene, ${ }^{43,44}$ circumcoro-graphyne, ${ }^{45}$ coro-graphene, ${ }^{45}$ Wsheet, ${ }^{42,46}$ and phagraphene. ${ }^{47,48}$ Very interesting it is the successful fabrication of graphdiyne on experiment, ${ }^{21,22}$ which shows the promise in experimentally producing new carbon sheet structures. Recently, Wang et al. ${ }^{47}$ proposed the phagraphene composed of 5-6-7 carbon polygons, which is lower in energy than most of the previously predicted $2 \mathrm{D}$ carbon allotropes. Also, considering the fact of the 5-7 polygons known as
Laboratory of Advanced Materials Physics and Nanodevices, School of Physics and Technology, University of Jinan, Jinan 250022, China. E-mail:phdgchen@hotmail.com $\dagger$ Electronic supplementary information (ESI) available. See DOI: 10.1039/c7ra01762f 
Stone-Wales defect ${ }^{49,50}$ is stable in the defected graphene, the phagraphene holds the promise for fabrication. In addition to the geometrical stability of the 5-7 polygons, the 5-8 polygons are actually also stable configuration as evidenced in the experimental studies of the 5-8 defect chain in graphene. ${ }^{51,52}$ By arranging the pentagons, hexagons, and octagons, Mandal et $a l .{ }^{31}$ and Sharma et $a l .{ }^{30}$ recently reported two different 2D carbon allotropes composed of the 5-6-8 carbon rings, which are referred in this paper as the $\gamma$ - and $\varepsilon$-phographenes (accounting the pentagon-hexagon-octagon structural characteristics) to facilitate discussion. By carrying out the global minimum structure search with the efficient evolutionary algorithm, we have found three new structural isomers of the phographene, which would be discussed as the $\alpha$-, $\beta$-, and $\delta$ phographenes in this paper. Among the structural isomers of phographene, the $\alpha$-phographene has the lowest energy. The previously reported $\gamma$ - and $\varepsilon$-phographenes are $\sim 50$ and $\sim 150$ meV per atom higher in total energy, suppressing their experimental fabrications. Furthermore, the $\alpha$-phographene is a little lower in energy than the recently reported 5-6-7 polygon characterized phagraphene, ${ }^{47}$ which may gain much lower total energy in the charged state. This in combination with our detailed studies of the dynamic, thermal, and mechanical stabilities suggest the priority of its experimental realization. Additionally, our studies on the electronic properties, the mechanical properties, the hydrogenation effects, and the usage in supporting precious metal clusters as complex catalyst nanostructures show attractive fundamental and application properties of the phographene, calling for further investigations on both theory and experiment.

\section{Computational details}

The first principles calculations were carried out with the density functional theory (DFT) as implemented in the Vienna $a b$ initio simulation package (VASP). ${ }^{53}$ Both the projector augmented-wave potential and the planewave basis set were used. ${ }^{54}$ The cutoff energy of the plane-wave basis set was set to $500 \mathrm{eV}$. The exchange and correlation energies were treated by using the generalized gradient approximation (GGA) with the Perdew, Burke, and Ernzerhof (PBE) parameterization. ${ }^{55}$ The phographene was placed in the $x y$ plane, with a vacuum of $15 \AA$ along $z$ direction to minimize the effects from its neighboring images. The Monkhorst-Pack technique ${ }^{56}$ was used to sample the $k$ points in Brillouin zone. According to the primitive unit cells for the planar $\alpha$-, $\beta$-, $\gamma$-, $\delta$ - and $\varepsilon$-phases, the $k$-meshes of 7 $\times 7 \times 1,5 \times 11 \times 1,9 \times 11 \times 1,5 \times 9 \times 1$, and $9 \times 13 \times 1$ were carefully tested. The energies were converged to $10^{-5} \mathrm{eV}$ and the calculated Hellmann-Feynman forces acting on each atom were converged to $20 \mathrm{meV} \AA^{-1}$. The phonon dispersion was calculated with the Phonopy code using the finite displacement method. ${ }^{57}$ In order to obtain reliable results, the density functional theory calculations with higher accuracy were performed, for which the criteria of $10^{-8} \mathrm{eV}$ and $0.1 \mathrm{meV}^{-1}$ were adopted for the convergences of the energies and the Hellmann-Feynman forces. Ab initio molecular dynamics simulations (AIMD) were carried out to estimate the thermal stability. Referring to the primitive unit cell, we used the $4 \times 4 \times 1$ sized supercell to minimize the effects from the periodic boundary conditions to explore the possible structural reconstruction. During the simulation, the temperature was controlled via the Nosé-Hoover technique. ${ }^{58}$ The global minimum structural search was carried out by using an efficient evolutionary algorithm as implemented in the Universal Structure Predictor: Evolutionary Xtallography (USPEX), ${ }^{59-62}$ which is interfaced with VASP code to select the low-energy structural configurations. Among the 17 plane space groups, the randomly selected 2D symmetry groups were adopted in generating geometrical structures of the $2 \mathrm{D}$ carbon structures. The structural searches started with the input carbon atoms ranging from 6 to 24, using the randomly generated lattice parameters and atomic coordinates.

\section{Results and discussion}

\subsection{Geometrical configuration and stability}

We began our study with a global minimum structural search using an efficient and systematic evolutionary structural search algorithm developed by Oganov and coworkers. ${ }^{5-62}$ In the evolutionary process of the structural search, the population of $30 \%$ of the structure candidates with low total energies were evolved over successive generations of random variation and selection. The structural evolutionary search with 20 carbon atoms in the unit cell is schematically shown in Fig. 1 for illustration. Besides the lowest energy honeycomb structure of graphene, both the previously reported phagraphene ${ }^{47}$ composed of 5-6-7 carbon rings and the carbon allotropes composed of 5-6-8 carbon rings were found as the low energy planar allotropes. In order to facilitate discussion, we would like to refer the 5-6-8 (penta-hexa-octa) carbon allotrope as phographene. In Fig. 2, we show the geometrical configurations of the phographenes and the corresponding phase diagram calculated on PBE-level. The corresponding lattice parameters are prvodied in Table 1 . The $\alpha$-, $\beta$-, and $\delta$-phases are the first time proposed carbon allotropes while the $\gamma$ - and $\varepsilon$-phases correspond to the previously reported ones by Mandal et al. ${ }^{\mathbf{3 1}}$ and Sharma et al.,$^{30}$ respectively. Referring to the lowest energy $\alpha$-phographene, the $\beta$-, $\gamma$-, $\delta$-, and $\varepsilon$-phases are calculated on PBE-level to be 7, 50, 64, and $157 \mathrm{meV}$ per atom higher in total energy, respectively. Besides the relative energies calculated with PBE functional, the corresponding energies calculated with the local density approximation (LDA), ${ }^{\mathbf{6 3} 64}$ Perdew-Wang 91 (PW91), ${ }^{65}$ Tao-Perdew-Staroverov-Scuseria (TPSS) ${ }^{66}$ and the

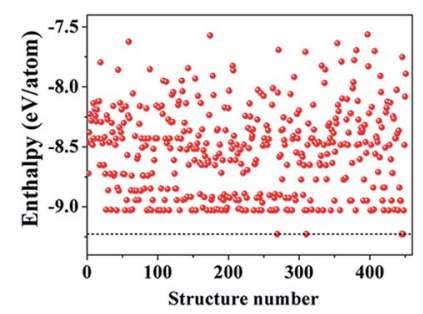

Fig. 1 Typical enthalpy evolution for the evolutionary structural search. 


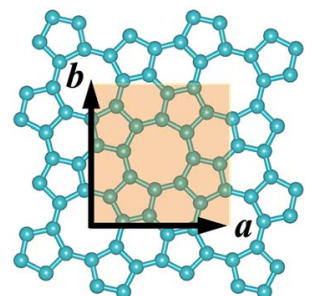

(a)

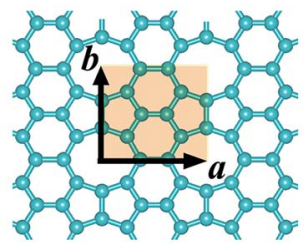

(c)

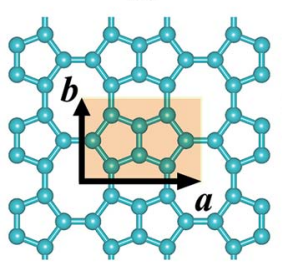

(e)

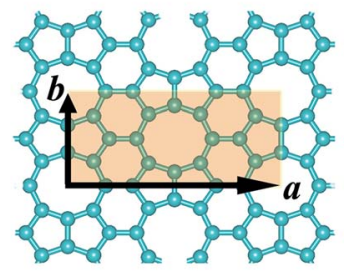

(b)

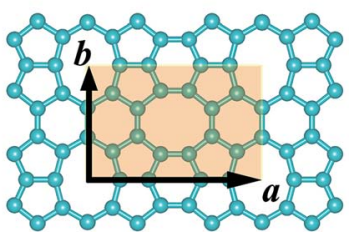

(d)

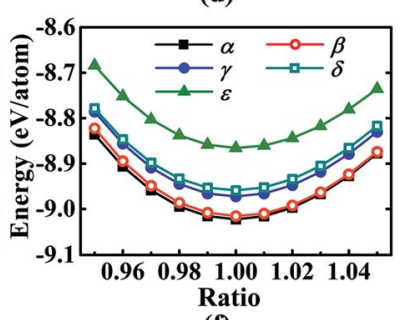

(f)

Fig. 2 Geometrical configurations in the (a), (b), (c), (d), and (e) are for the $\alpha^{-}, \beta^{-}, \gamma^{-}, \delta_{-}^{-}$, and $\varepsilon$-phographenes, respectively. The corresponding primitive unit cells are highlighted by the light-brown shaded areas. The cyan balls stand for the carbon atoms. The phase diagrams of the studied phographenes are shown in (f).

Table 1 The lattice constants $(\AA)$ of the primitive unit cells $a \times b$ and the corresponding numbers $N$ of the carbon atoms contained in the unit cells. The primitive unit cells of the studied phographenes are shown in Fig. 2

\begin{tabular}{llllll}
\hline Parameter & $\alpha$ & $\beta$ & $\gamma$ & $\delta$ & $\varepsilon$ \\
\hline$a$ & 7.42 & 11.14 & 5.61 & 8.66 & 5.79 \\
$b$ & 7.42 & 4.91 & 4.89 & 5.71 & 3.83 \\
$N$ & 20 & 20 & 10 & 18 & 8
\end{tabular}

revised TPSS meta-GGA (rev-TPSS) ${ }^{67}$ are also tabulated in Table 2 . The newly proposed $\alpha$-phographene is almost degenerate in energy with the previously reported phagraphene. The in-plane densities of the carbon atoms of the $\alpha$ - and $\beta$-phographenes and the phagraphene ${ }^{47}$ are $0.363,0.366$, and 0.371 atoms per $\AA^{2}$. The $\alpha$-phographene with smaller atomic density is found to have the lowest energy, hinting the effects of the topological arrangement of the carbon polygons. The other new carbon allotropes $\beta$ - and $\delta$-phographenes are about 7 and 64 meV per atom higher in energy. The previously reported $\gamma$ - and $\varepsilon$-phographenes have about 51 and $156 \mathrm{meV}$ per atom higher energies. The other carbon allotropes as the biphenylene, ${ }^{26}$ OPG-L ${ }^{43}$ OPG-Z ${ }^{43}$ circumcoro-graphyne, ${ }^{45}$ coro-graphene, ${ }^{45} \mathrm{C}_{4}$ sheet, ${ }^{36-42}$ graphenylene,$^{24,25}$ graphyne,${ }^{10-20}$ graphdiyne ${ }^{20-23}$ and penta-graphene ${ }^{29}$ are found to be about 103, 112, 148, 129, 231, $307,427,502,555$, and $708 \mathrm{meV}$ per atom higher in energy,

Table 2 The relative energies (meV per atom) calculated on different theoretical levels of the 2D carbon allotropes as referred to the lowest energy one. Besides the newly proposed $\alpha-, \beta-$, and $\delta$-phographenes, the previously reported $\gamma^{-31}$ and $\varepsilon$-phographenes, ${ }^{30}$ phagraphene, ${ }^{47}$ biphenylene, ${ }^{26}$ OPG-L and OPG- $Z,{ }^{43}$ circumcoro-graphene (CCG), ${ }^{45}$ coro-graphene $(\mathrm{CG})_{,}{ }^{45} \mathrm{C}_{4}$ sheet, $^{36-42}$ graphenylene, ${ }^{24,25}$ graphdiyne, ${ }^{21-23}$ graphyne, ${ }^{10-20}$ and penta-graphene ${ }^{29}$ are also calculated for comparison

\begin{tabular}{lrrrrr}
\hline Allotrope & LDA & PW91 & PBE & TPSS & rev-TPSS \\
\hline Phagraphene & 0.0 & 0.0 & 0.4 & 1.8 & 1.9 \\
$\alpha$-Phographene & 5.9 & 0.4 & 0.0 & 0.0 & 0.0 \\
$\beta$-Phographene & 10.6 & 7.5 & 6.8 & 7.7 & 6.9 \\
$\gamma$-Phographene & 54.5 & 50.9 & 50.3 & 51.8 & 51.2 \\
$\delta$-Phographene & 67.9 & 64.4 & 63.5 & 64.5 & 62.4 \\
$\varepsilon$-Phographene & 157.4 & 157.6 & 156.7 & 155.5 & 152.2 \\
Biphenylene & 112.4 & 104.4 & 103.3 & 102.8 & 102.4 \\
OPG-L & 122.9 & 114.0 & 112.3 & 112.3 & 110.7 \\
OPG-Z & 158.0 & 149.3 & 147.6 & 148.3 & 146.1 \\
CCG & 143.5 & 131.7 & 132.5 & 125.2 & 126.3 \\
CG & 251.4 & 235.9 & 234.1 & 226.7 & 226.3 \\
C 4 sheet & 337.8 & 310.1 & 315.7 & 298.7 & 301.6 \\
Graphenylene & 461.6 & 429.9 & 426.5 & 426.4 & 424.4 \\
Graphdiyne & 676.5 & 557.2 & 564.6 & 538.4 & 558.5 \\
Graphyne & 594.2 & 502.3 & 508.6 & 489.7 & 505.5 \\
Penta-graphene & 691.0 & 709.0 & 704.0 & 718.8 & 698.4
\end{tabular}

respectively. Based on the analyses on the calculated energies, we would like hereafter to concentrate on discussing the newly proposed $\alpha$ - and $\beta$-phographenes. At the end of the section of the discussion and results, we would also attempt to discuss the possibilities of their fabrications.

The dynamic and thermal stabilities of the $\alpha$ - and $\beta$-phographenes are studied in Fig. 3. The calculated phonon spectrum
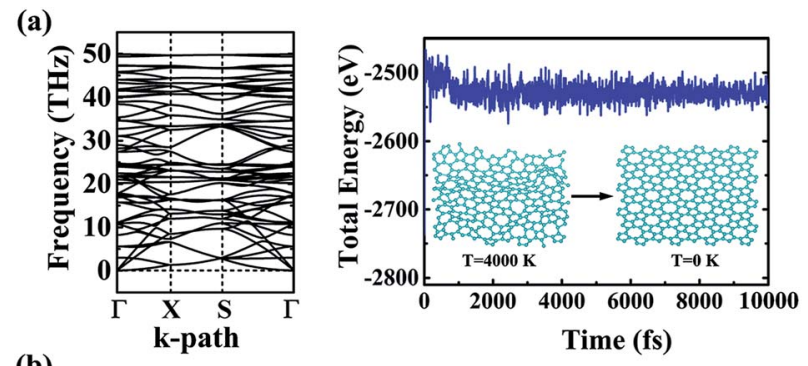

(b)
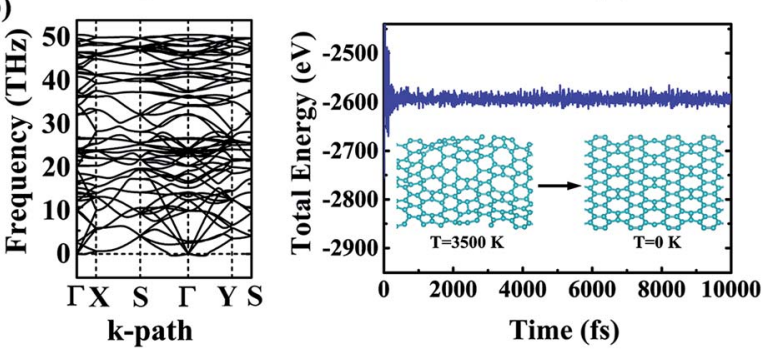

Fig. 3 The phonon spectra (left) and the total energies as a function of the time of the AIMD simulations (right). The (a) and (b) are for the $\alpha$ and $\beta$-phographenes, respectively. The insets show the structures obtained at the end of simulations and the corresponding structures fully optimized at $0 \mathrm{~K}$. 
of $\alpha$-phographene does not have any imaginary mode in the entire Brillouin zone, confirming its dynamic stability. However, the phonon spectrum shows the almost neglected imaginary modes round $\Gamma$ point for the $\beta$-phographene. Considering the good stabilities judged from our later discussion of the AIMD simulations and the mechanical stability evaluation, the presence of the imaginary modes may be attributed to the uncertainties of our calculations. Also, these imaginary modes would be easily removed by applying a slight tensile strain on the $\beta$-phographene. In Fig. 3, we have carefully evaluated the melting temperature of the $\alpha$ - and $\beta$-phographenes to estimate their heat stabilities by using the AIMD simulations, which have been confirmed to be useful in evaluating the thermal stabilities of planar materials. ${ }^{68,69}$ In order to minimize the constraints of the periodic boundary conditions on the atomic movement, the carbon allotropes were simulated by using the $4 \times 4 \times 1$ supercells for exploring possible structural reconstructions. The geometrical structures were monitored during the simulations. The stabilities of the corographene and circumcoro-graphyne ${ }^{45}$ were previously estimated at $300 \mathrm{~K}$, which are evaluated at $1000 \mathrm{~K}$ of the phagraphene $^{47}$ and penta-graphene. ${ }^{29}$ In our studies, we have estimated the highest temperatures for the $\alpha$ - and $\beta$-phographenes to withstand against structural reconstructions, which are found to be 4000 and $3500 \mathrm{~K}$, respectively. In Fig. 3, the evolutions of their total potential energies as a function of simulation time for the AIMD simulations at the corresponding highest temperatures are shown, respectively. Additionally, the structures obtained at the end of the simulations are presented as the insets in Fig. 3, which are 0.63 and $0.49 \mathrm{eV}$ per atom higher in energy than the ideal planar structures of the corresponding ground states. Although structural distortions could be clearly seen, the structural skeletons are in fact preserved. Using them as the initial states, we have also carried out full structural optimizations at $0 \mathrm{~K}$, which could quickly converge to the corresponding ground states, suggesting $\alpha$ - and $\beta$-phographenes to be protected by high energy barriers against structural transitions on the potential energy surface.

As to the application, the strain may also happen to the carbon allotrope, for example, which could be induced by the lattice mismatch between the planar sheet and the supporting substrate. Therefore, the mechanical stabilities of $\alpha$ - and $\beta$ phographenes are also worth of evaluating. In Fig. 4, we have studied the strain energy versus the applied strain, which is calculated by subtracting the total energy of the ground state structure from that of the strained structure. The Fig. 4a and e show the results of the $\alpha$ - and $\beta$-phographenes under the equibiaxial and uniaxial strains, respectively. The corresponding energy surfaces versus the strains applied along the arbitrary inplane directions are provided in Fig. $4 \mathrm{~b}$ and f, respectively. The smooth evaluations of strain energies show that the deformation energies keep positive in the range of the studied strain loading in Fig. 4b and $\mathrm{f}$, which suggest the mechanical stabilities according to the mechanical stability criteria. ${ }^{8}$ Additionally, we have calculated the corresponding linear elastic constants by using the finite distortion method. Following the standard Voigt notation, the elastic strain energy per unit can be written as:
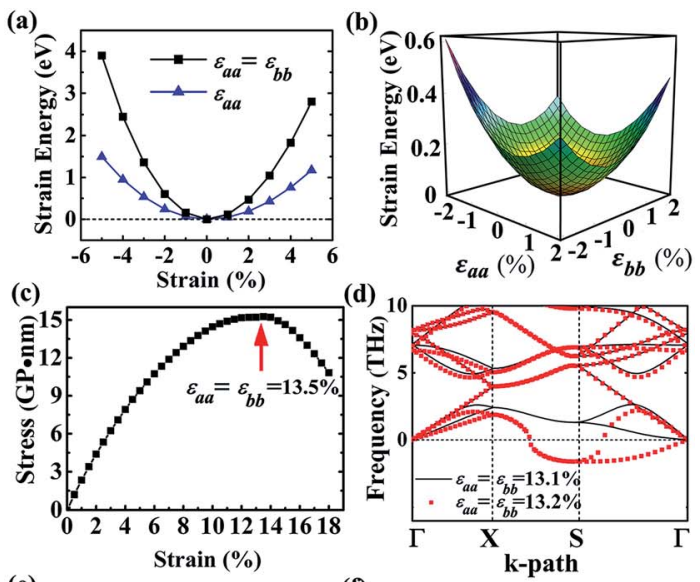

(e)
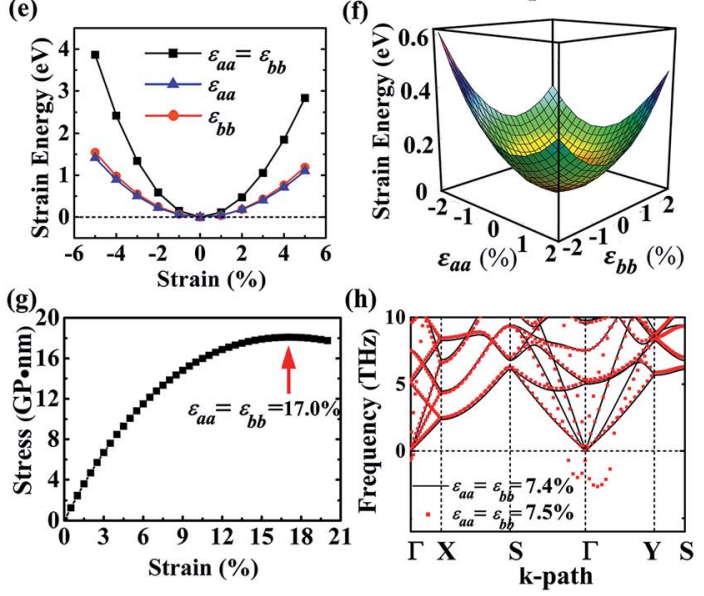

Fig. 4 The (a)-(d) and (e)-(h) show the mechanical stabilities of the $\alpha$ and $\beta$-phographenes, respectively. The (a) and (e) are the energy increases induced by the uniaxial and equi-biaxial in-plane strains. And, the corresponding energy surfaces versus the arbitrary in-plane strains are shown in (b) and ( $\mathrm{f}$ ). The stress-strain relationships ( $c$ and $\mathrm{g}$ ) and the phonon spectra ( $d$ and $h$ ) of the equi-biaxially strained phographenes are also shown.

$$
U(\varepsilon)=\frac{1}{2} C_{11} \varepsilon_{\mathrm{aa}}^{2}+\frac{1}{2} C_{22} \varepsilon_{\mathrm{bb}}^{2}+C_{12} \varepsilon_{\mathrm{aa}} \varepsilon_{\mathrm{bb}}+2 C_{66} \varepsilon_{\mathrm{ab}}^{2}
$$

where $\varepsilon_{\mathrm{aa}}$ and $\varepsilon_{\mathrm{bb}}$ are the uniaxial strains respectively applied along $a$ and $b$ directions, and $\varepsilon_{\mathrm{ab}}$ is the equi-biaxial strain. $C_{11}$, $C_{22}, C_{12}$, and $C_{66}$ are the components of elastic modulus tensor, which could be obtained by calculating the second partial derivative of strain energy with respect to strain. ${ }^{29}$ In order to validate the accuracy of our method, the elastic constants of the graphene sheet were firstly computed. We got the data of $C_{11}=$ $C_{22}=346.3$ and $C_{12}=C_{21}=71.8 \mathrm{~N} \mathrm{~m}^{-1}$, in agreement with the experimental measurements ${ }^{70}$ and the previous computations. ${ }^{68}$ For the carbon allotropes $\alpha$ - and $\beta$-phographenes, the calculated elastic constants are tabulated in Table 3. One can see that both of them have the $C_{11} C_{22}-C_{12}^{2}>0$ and $C_{66}>0$, satisfying the Born-Huang mechanical stability criteria. ${ }^{71}$ Besides, we have estimated the maximum equi-biaxial strain that the phographene could withstand. The results of the $\alpha$ - and $\beta$-phographenes as presented in Fig. $4 \mathrm{c}$ and $\mathrm{g}$ show that the maximum values happen at the $13.5 \%$ and $17.0 \%$, respectively, hinting 
Table 3 The calculated elastic constants of the $\alpha$ - and $\beta$ phographenes

\begin{tabular}{lllll}
\hline Phographene & $C_{11}$ & $C_{22}$ & $C_{12}$ & $C_{66}$ \\
\hline$\alpha$ & 310.41 & 310.41 & 79.65 & 130.63 \\
$\beta$ & 320.41 & 293.58 & 141.86 & 95.64
\end{tabular}

good mechanical stabilities. Considering the facts that the dynamic effects may worse the stability of the strained sheet material, ${ }^{72}$ we also analyzed the corresponding phonon spectra of the phographenes after applying the equi-biaxial strains. The calculated phonon spectra presented in Fig. $4 \mathrm{~d}$ and $\mathrm{h}$ show that the maximum values before structural collapse occurring are reduced to $13.1 \%$ and $7.4 \%$ for the $\alpha$ - and $\beta$-phographenes, respectively. The $\alpha$-phographene shows better mechanical stability than the $\beta$-phographene.

Providing to use the new carbon allotrope in air conditions, the structural stability may bear effects from the oxygen. In our studies, we have carefully studied the adsorption of an $\mathrm{O}_{2}$ molecule on the phographenes. Same as to the hexagonal graphene, both the $\alpha$-phographene and the $\beta$-phographene could not adsorb the $\mathrm{O}_{2}$, hinting the structural stability for the applications in air conditions. Additionally, hydrogenation is the often used technique for modifying the properties of $2 \mathrm{D}$ materials. We have also evaluated the stabilities of the hydrogenated $\alpha$ - and $\beta$-phographenes, whose lowest energy configurations are schematically shown in Fig. 5 along with the corresponding phonon spectra (the higher energy hydrogenation configurations are provided in the ESI Fig. S1 and S2 $\dagger$ ). In comparison with the phonon spectra for the pristine phographenes shown in Fig. 3, the presence of the high frequency (a)

(b)

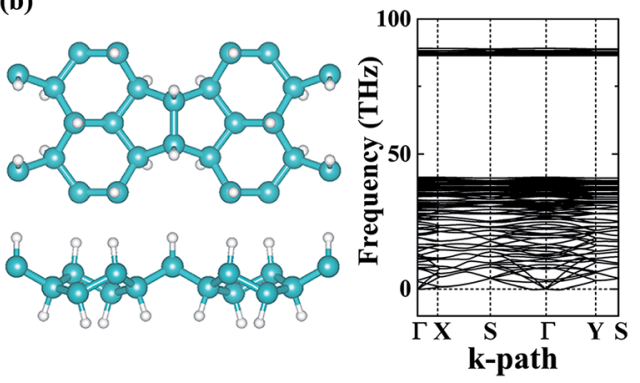

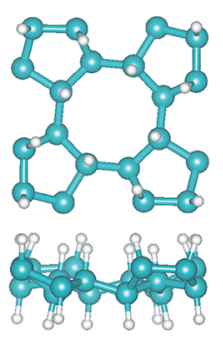

Fig. 5 The lowest energy configurations of the hydrogenated phographenes and the corresponding phonon spectra. The (a) and (b) are for the $\alpha$ - and $\beta$-phographenes, respectively. The cyan and white balls stand for the carbon and hydrogen atoms, respectively. modes comes from the covalent $\mathrm{C}-\mathrm{H}$ bonding. Also, no imaginary mode in the simulated spectra suggests the structural stabilities.

\subsection{Mechanical and electronic properties}

Considering the applications, the mechanical properties of the carbon allotropes are also worth of evaluating on theory. Based on the tensor components calculated for the eqn (1), we studied the in-plane Young's modulus for the $\alpha$ - and $\beta$-phographenes, which are shown in Fig. 6 . The $0^{\circ}, 45^{\circ}$, and $90^{\circ}$ angles correspond to the directions along with the lattice $a$, the diagonal axis, and the lattice $b$ of the corresponding primitive unit cells, respectively. For the $\alpha$-phographene, the lattices $a$ and $b$ are symmetrically equivalent resulting in the same Young's moduli along them, which are calculated to be $290.0 \mathrm{~N} \mathrm{~m}^{-1}$. As shown in Fig. 6, the modulus along with the directions corresponding to the angles between $0^{\circ}$ and $90^{\circ}$ would get larger. The largest Young's modulus is found to be in the diagonal direction (corresponds to the $45^{\circ}$ angle), which is $312.9 \mathrm{~N} \mathrm{~m}^{-1}$. The $\beta$ phographene has different conditions, whose modulus along the lattices $a$ and $b$ are calculated to be 251.9 and $230.8 \mathrm{~N} \mathrm{~m}^{-1}$, respectively. The maximum is also found in the diagonal direction, which is $268 \mathrm{~N} \mathrm{~m}^{-1}$. Both of the primitive unit cells of them contain $20 \mathrm{C}$ atoms, respectively. The areas of the $2 \mathrm{D}$ unit cells are 55.1 and $54.7 \AA^{2}$ for the $\alpha$ - and $\beta$-phographenes, respectively. However, the calculated in-plane Young's moduli show that the $\beta$-phographene with slightly densely arranged C atoms is softer than the $\alpha$-phographene, bearing the effects from the topological arrangements of the penta-, hexa-, and octa-carbon rings. Additionally, the Young's moduli of graphene,${ }^{4}$ phagraphene, ${ }^{47}$ and penta-graphene ${ }^{29}$ are calculated to be $331.4,292.9,263.8 \mathrm{~N} \mathrm{~m}^{-1}$, respectively. The $\alpha$-phographene shows comparable in-plane stiffness with graphene, suggesting good mechanical properties. In Fig. 6, the Poisson's ratio, $\nu$, has also been theoretically estimated. The stiffer $\alpha$-phographene shows overall smaller ratios as compared with the $\beta$-phographene. In comparison with the $\nu=0.21$ referring to the

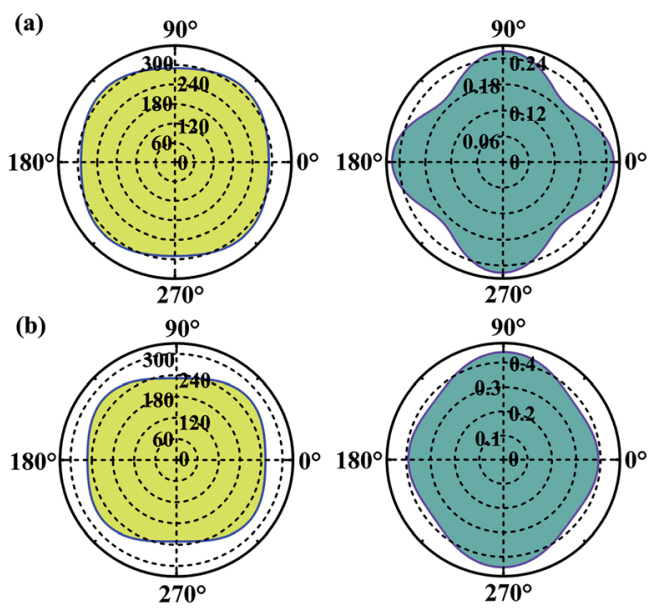

Fig. 6 The orientation-dependences of the Young's modulus in $\mathrm{N} \mathrm{m}^{-1}$ (left) and Poisson's ratio (right) of the $\alpha$ - and $\beta$-phographenes, respectively. 
stretching in the lattice direction of graphene, the studied Poisson's ratios also show remarkable mechanical properties of the $\alpha$-phographene. If the $\alpha$-phographene was stretched, it would act similarly with its cousin part graphene which would only shrink slightly in the lateral direction. The maximum and minimum values happen to the situations of stretching the $\alpha$ phographene along the lattice direction and the diagonal direction, which are $\sim 0.26$ and 0.20 , respectively. The maximum and minimum values of the $\beta$-phographene would be obtained by stretching it along the lattice $b$ and $a$ directions, which are calculated to be 0.44 and 0.38 , respectively. Again, the $\alpha$-phographene shows superior mechanical properties than the $\beta$-phographene.

The calculated energy bandstructures are presented in Fig. 7 for the $\alpha$ - and $\beta$-phographenes. Unlike graphene, no Dirac electronic characteristics are found. Both of them show metallic conducting properties. The effects of different topological arrangements of $\mathrm{C}$ atoms in the $2 \mathrm{D}$ carbon allotropes could also be seen in the corresponding conducting properties. Previous studies show that the graphene, ${ }^{\mathbf{4}}$ OPG-Z, ${ }^{\mathbf{4 3}}$ phagraphene, ${ }^{\mathbf{4 7}}$ and circumcoro-graphyne $^{\mathbf{4 5}}$ have Dirac cones showing semimetal properties. The graphyne, ${ }^{\mathbf{1 0}-20}$ graphdiyne,$^{\mathbf{2 0 - 2 3}}$ graphenylene,${ }^{\mathbf{2 4}}$ coro-graphene, ${ }^{45}$ and penta-graphene ${ }^{29}$ are semiconductors. And, the radialenes, ${ }^{28}$ pentahexoctite, ${ }^{30}$ HOP-graphene, ${ }^{31}$ pentaheptities, ${ }^{32,33}$ haeckelites, ${ }^{34}$ squarographene, ${ }^{35} \mathrm{C}_{4}$ sheet, ${ }^{36-42} \mathrm{~W}$ sheet, ${ }^{42,46}$ and OPG- $\mathrm{L}^{\mathbf{4 3 , 4 4}}$ are reported to be metallic. It is well known that the DFT with GGA-PBE has the deficiency in describing bandgap in the calculated bandstructure. It reduces the original problem to an auxiliary problem involving fictitious noninteracting particles moving in a one-body effective potential, the familiar Hartree potential, and an exchange-correlation
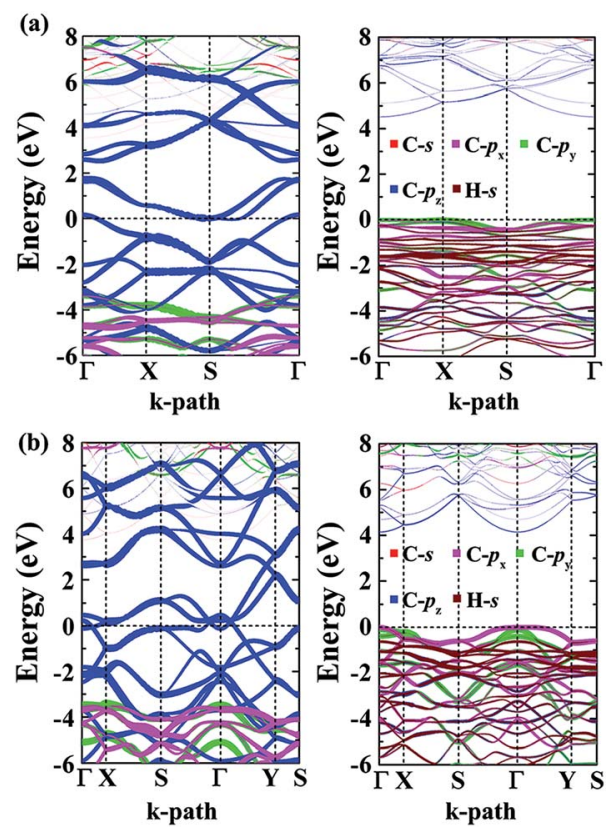

Fig. 7 The orbital-resolved bandstructures of the phographenes (left) and the corresponding hydrogenated sheets (right). The $\alpha$ - and $\beta$ phographenes are studied in the (a) and (b), respectively. potential. The byproduct of the Kohn-Sham approach is a set of single-particle eigenvalues, which tends to underestimate the bandgap. In this study, we have also applied the more accurate Heyd-Scuseria-Ernzerhof (HSE) hybrid functional which includes a screened short-range Hartree-Fock exchange term. ${ }^{73,74}$ The HSE06 functional with the empirical parameter $\alpha=0.25$ was adopted, which is widely used for studying the semiconductor materials. Our calculations on HSE level confirm the conducting properties of the $\alpha$ - and $\beta$-phographenes. One can see that the bandstructures shown in Fig. 7 have distinct characteristics for the $\alpha$ - and $\beta$-phographenes. There are nearly parallel bands in the proximity of Fermi energy of the $\alpha$-phographene to help increase the joint density of states at the energy of $\sim 1.6 \mathrm{eV}$, which may find applications in photoelectronics.

As shown in Fig. 7, both the valence and conducting bands around Fermi energy originate from the $\mathrm{p}_{z}$ orbitals in the $\alpha$-and $\beta$-phographenes, which would form the $\pi$-like bonds on both sides of the $2 \mathrm{D}$ carbon allotropes, agreeing with the $\mathrm{sp}^{2}$-like electronic hybridization configurations. In the fully hydrogenated allotropes, the $\mathrm{p}_{z}$ orbital would be saturated with hydrogen atoms. All of the electrons would be bound in the strong $\sigma$-like covalent bonds, opening wide bandgaps in the calculated bandstructures of the hydrogenated $\alpha$ - and $\beta$-phographenes. Previously, Sun and coworkers reported that the remarkable ferromagnetic properties could be obtained in the semi-hydrogenated graphene. ${ }^{75}$ As to the semi-hydrogenated phographenes, our careful analyses of the phonon spectra support their structural stabilities. Unlike the semihydrogenated graphene, the ground states of the hydrogenated phographenes are antiferromagnetic with more than $19 \mathrm{meV}$ per coupling than the corresponding ferromagnetic states, respectively. In the lowest energy configuration of the semihydrogenated phographene, the naked $\mathrm{C}-\mathrm{C}$ dimer pair gains presence in the pentagonal carbon rings, which tends to form $\pi$-like bond paring the corresponding $\mathrm{p}_{z}$ electrons. The unpaired $\mathrm{p}_{z}$ electrons are distributed on 4 carbon atoms in the carbon octagon, which are separated with each other by the carbon atoms bonded with $\mathrm{H}$ atoms on the octagonal ring, preferring the antiferromagnetic couplings.

\subsection{Discussion on the structural growth}

As shown in Table 2 , the newly proposed $\alpha$ - and $\beta$-phographenes are almost degenerate with the previously reported phagraphene in energy. From the point of view of the energy, the phagraphene and the low-lying phographenes gain almost the same possibilities in structural growing, challenging the experimental fabrications of $\alpha$ - or $\beta$-phographene single crystal materials. In experimental studies, the metal surfaces, such as the $\{111\}$-faceted surfaces of $\mathrm{Cu}, \mathrm{Ag}, \mathrm{Au}$, and $\mathrm{Pt}$, are usually used as substrates for growing $2 \mathrm{D}$ materials, which are rich of electrons in surface states. Furthermore, the methodology of nearsurface alloy could be used to alter the reactive of metal surfaces, ${ }^{76,77}$ which could be used to enhance the charge transfer between the metal substrate and its supported 2D materials. Additionally, the injection of electrons was previously used to 
engineer the reactivity of $2 \mathrm{D}$ materials. ${ }^{78}$ Therefore, we would like to consider the effects of the electron injection on the structural growth of carbon allotropes, with the purpose to shed light on the corresponding experimental fabrications. In Fig. 8a, the energy evolutions of the low energy carbon allotropes are studied as a function of the quantity of the injected electrons. Among the examined allotropes, the $\alpha$ - and $\beta$-phographenes could be separated from the other ones, which would get much more favored for experimental fabrications according to the lower total energies in the charged states. For example, the phagraphene would be $\sim 60 \mathrm{meV}$ per atom higher than the $\alpha$ and $\beta$-phographenes in the -0.2 e per atom charged state, which are however almost degenerate in energy in the neutral state.

From the point of view of the energy, we reach now that the $\alpha$ - and $\beta$-phographenes could be not separated with each other in both neutral and negatively charged states. Though the positively charged state may have difficulty to be realized on experiment, we also calculated the energies of the $\alpha$ - and $\beta$ phographenes after removing some electrons from the corresponding neutral states, which however could not bring obvious energy difference between them also. In order to shed light on the possibilities of which phase of phographene would gain priority for fabrication, we also studied the formation of the corresponding structural units. The pentagonal, hexagonal, heptagonal, and octagonal rings are the structural units which could be obtained on experiment for carbon nanostructures. We start our studies with an isolated carbon octagon, followed

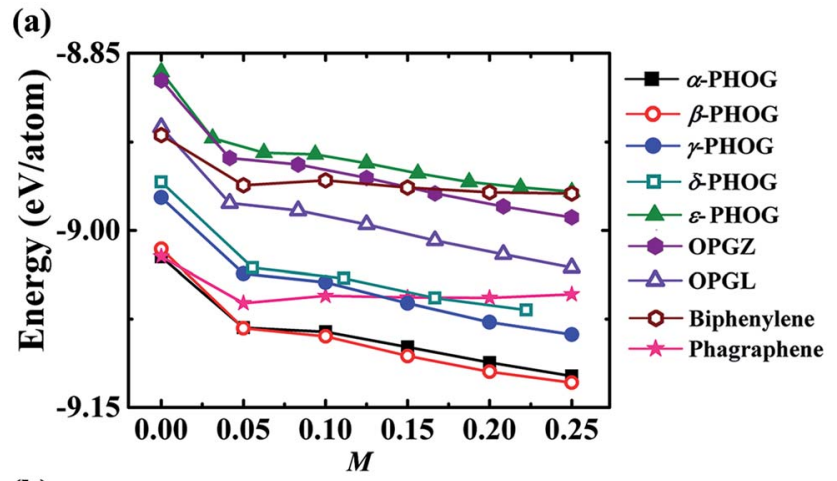

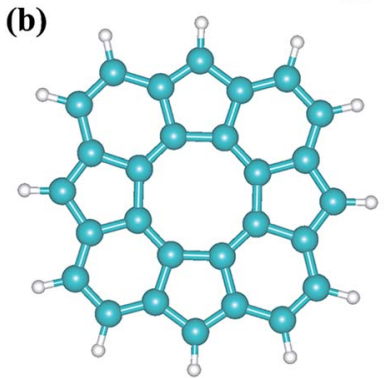

$0.00 \mathrm{eV}$

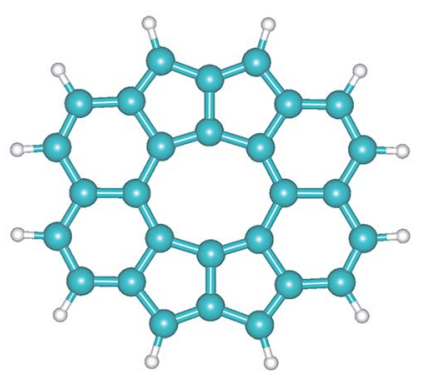

$1.12 \mathrm{eV}$
Fig. 8 (a) The calculated total energy (eV per atom) as a function of the amount $M$ of the injected electrons. (b) The structural motifs of the $\alpha$ and $\beta$-phographenes. The motif of the $\beta$-phographene is $1.12 \mathrm{eV}$ higher in total energy than the one of the $\alpha$-phographene. The cyan and white balls stand for the carbon and hydrogen atoms, respectively. by introducing more carbon rings (see the ESI Fig. S3 $\dagger$ ). Based on the carbon octagon, the formation of an adjacent carbon hexagon is a little lower in energy than a carbon pentagon. Introducing one more carbon ring, the configuration of the hexagonal and pentagonal rings adhering on the adjacent $\mathrm{C}-\mathrm{C}$ edges of the seed structural unit - the carbon octagon and sharing one $\mathrm{C}-\mathrm{C}$ between them would be preferable. Again, adding one more carbon ring, the lowest energy structure shows the tendency for arranging the hexagonal and pentagonal rings alternatively on the adjacent $\mathrm{C}-\mathrm{C}$ edge of the carbon octagon. Following this trend, the structural fragment of the $\alpha$-phographene could be obtained (see the Fig. 8b). In comparison, the structural fragment of the $\beta$-phographene shown in Fig. $8 \mathrm{~b}$ is also studied. While both the studied fragments contain $20 \mathrm{C}$ atoms and $12 \mathrm{H}$ atoms, the one for the $\alpha$-phographene gains $1.12 \mathrm{eV}$ lower total energy to gain priority for experimentally synthesis. This may facilitate the fabrication of the $\alpha$-phographene, calling for experimental realization.

\subsection{Catalytic properties as supporting noble metal catalysts}

Interestingly, a most recent theory-experiment joint study shows the advances in achieving high-performance catalytic properties by using the nitrogen-doped graphene to support platinum atoms or particles, where the enhanced interaction between $\mathrm{Pt}$ and $\mathrm{N}$-doped graphene plays an important role. ${ }^{79} \mathrm{In}$ the ideal pristine graphene, the $\mathrm{p}_{z}$ electrons would be bound in the conjugate $\pi$-bonds making the graphene to some sense inert, whose reactivity could be enhanced by the structural defect, chemical modification, bending curvature, and tensile strain, etc. ${ }^{\mathbf{8 0 - 8 4}}$ Compared with the graphene, the $\mathrm{sp}^{2}$ hybridization in phographene is perturbed, which may in turn also affect the reactivity. Hereafter, with the purpose to facilitate discussion and considering the above discussion on the structural growth, we would like to take the $\alpha$-phographene as the prototype structure for examining the topologically enhanced reactivity. In our studies, we have calculated the adsorption of the $\mathrm{O}_{2}, \mathrm{H}_{2}, \mathrm{CO}$, and $\mathrm{N}_{2}$ gas molecules on the $\alpha$-phographene, which would not be hold in chemisorption states. However, the difference in reactivity between graphene and phographene could be seen in the adsorption of Au atom. In our studies, we have calculated the adsorptions of $\mathrm{Au}$ and Pt clusters with the PBE functional. The relativistic effects were included by using the scalar relativistic approach. A single Au atom could not be adsorbed on graphene while it can adhere on phographene by developing chemical-like bonding between them. The best adsorption site is upon a carbon atom (see Fig. 9a). The bond length between $\mathrm{Au}$ and $\mathrm{C}$ atoms is $2.19 \AA$, being slightly longer than the corresponding atomic radius sum (2.05 $\AA$ ). Additionally, the adsorption energy, $E_{\mathrm{ad}}$, is calculated to be $0.43 \mathrm{eV}$ by the below definition.

$$
E_{\text {ad }}=E_{\text {sheet }}+E_{\text {metal }}-E_{\text {metal } / \text { sheet }}
$$

where the $E_{\text {sheet }}, E_{\text {metal }}$, and $E_{\text {metal/sheet }}$ are the calculated total energies of the free-standing carbon sheet, the studied metal species, and the metal adsorbed carbon sheet. Also, compared to the adsorption on graphene, a Pt atom could be bound more 
(a)
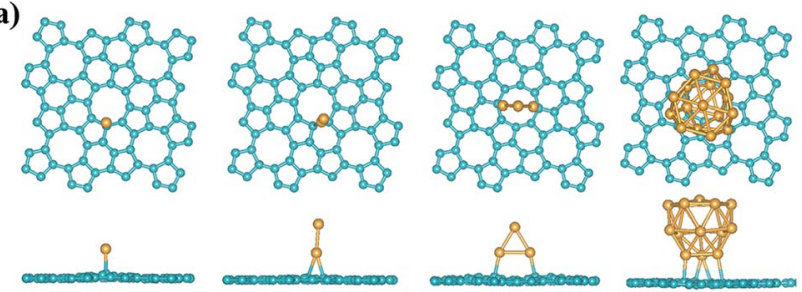

(b)
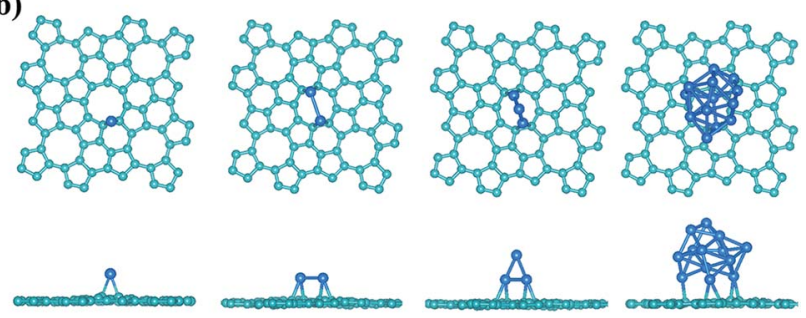

Fig. 9 The geometrical configuration of the Au (a) and Pt (b) clusters adsorbed on the $\alpha$-phographene. The cyan, golden, and azure balls stand for the $\mathrm{C}, \mathrm{Au}$, and Pt atoms, respectively. Both top and side views are provided.

strongly on phographene. The adsorption energies of a single Pt adsorbed on graphene and phographene are calculated to be 1.58 and $2.16 \mathrm{eV}$, respectively. The adsorption is upon the bridge site of a C-C bond (see Fig. 9b). On the graphene doped with a $\mathrm{N}$ atom, the lowest energy adsorption site for Pt atom is on the bridge site upon the $\mathrm{C}-\mathrm{C}$ bond adjacent to the $\mathrm{N}$ dopant. The presence of $\mathrm{N}$ makes its nearby $\mathrm{C}$ atoms more reactive to enhance the adsorption energy to $1.92 \mathrm{eV}$. One can see that the binding of Pt on the $\alpha$-phographene is a little stronger than that on the $\mathrm{N}$ doped graphene. Additionally, we have also studied the dimers and trimers of $\mathrm{Au}$ and $\mathrm{Pt}$, whose adsorption energies on both graphene and phographene allotropes are tabulated in Table 4. The bigger size clusters of the $\mathrm{Au}_{16}$ with hollow cage structure ${ }^{85,86}$ and the $\mathrm{Pt}_{13}$ with icosahedral compact structure ${ }^{87}$ are also used as prototypes to shed light on the adsorptions of noble metal clusters on the phographene. As seen in Table 4, though the $\mathrm{Au}_{2}, \mathrm{Au}_{3}$, and $\mathrm{Au}_{16}$ clusters could not be adsorbed on graphene, they would like to adhere to the phographene allotrope, suggesting the enhanced activities of the phographene. As compared to the adsorptions on the graphene, the binding strengths of the Pt clusters are also significantly enhanced. Similar as the complex catalyst nanostructures of the dispersedly adsorbed Pt clusters on the $\mathrm{N}$ doped graphene, the phographene allotrope may also gain preference in dispersedly supporting noble metal clusters as complex catalyst nanomaterials. With the purpose to shed light on the catalytic properties of the adsorbed noble metal clusters, we have

Table 4 The adsorption energies (eV) of the precious metal clusters adsorbed on the graphene and $\alpha$-phographene

\begin{tabular}{lllllllll}
\hline Structure & $\mathrm{Au}$ & $\mathrm{Au}_{2}$ & $\mathrm{Au}_{3}$ & $\mathrm{Au}_{16}$ & $\mathrm{Pt}$ & $\mathrm{Pt}_{2}$ & $\mathrm{Pt}_{3}$ & $\mathrm{Pt}_{12}$ \\
\hline Graphene & - & - & - & - & 1.59 & 0.96 & 1.44 & 0.57 \\
Phographene & 0.43 & 0.73 & 1.18 & 0.31 & 2.16 & 1.68 & 2.67 & 1.72
\end{tabular}

examined the adsorption of the $\mathrm{O}_{2}$ molecule. It would be adsorbed in chemisorption states with the $\mathrm{O}-\mathrm{O}$ bond being parallel adhered upon a Au-Au or Pt-Pt bonds. Referring to the ${ }^{3} \sum_{\mathrm{g}}{ }^{-}$ground state $\mathrm{O}_{2}$, the lengths are elongated of $\sim 13 \%$ and $11 \%$ on average of the $\mathrm{O}_{2}$ molecules adsorbed on the studied $\mathrm{Au}$ and Pt clusters, respectively, being in activated state with enhanced reactivity for oxidizing $\mathrm{CO}$ etc. ${ }^{82,88}$ To conclude, our results on the adsorptions of the Pt and Au species show the promise to anchor precious noble metal atoms or clusters on phographene to design high-performance catalyst, calling for further experimental investigations for realization.

\section{Conclusions}

Based on the density functional theory computation and the global minimum structural search, we have found the $\alpha$-, $\beta$-, and $\delta$-phographenes as new carbon allotropes. The $\alpha$-phographene is almost degenerate in energy with the previously reported phagraphene. The $\beta$ - and $\delta$-phographenes are about 7 and 63 meV per atom higher in energy. The previously reported $\gamma$ - and \&-phographenes have about 51 and $155 \mathrm{meV}$ per atom higher energies. The other known non-honeycomb 2D carbon allotropes including the graphyne, graphdiyne, $\mathrm{C}_{4}$-sheet, pentagraphene, etc. are $>100 \mathrm{meV}$ per atom higher in energy. Both the $\alpha$ - and $\beta$-phographenes are found to have superior dynamic, thermal, and mechanical stabilities. In the charged state, the $\alpha$ and $\beta$-phographenes would be significantly lowered in energy as compared to the phagraphene. Furthermore, our analysis on the growth of structural motifs shows the preference of the fabrication of $\alpha$-phographene. Additionally, the $\alpha$-phographene has close Young's modulus and Poisson's ratio to those of graphene, indicating remarkable mechanical properties. Both $\alpha$ - and $\beta$-phographenes are metallic. The $\alpha$-phographene shows enhanced joint density of states at the energy of $\sim 1.6 \mathrm{eV}$, which may find applications in photoelectronics. Hydrogenation would alter them to wide-gap semiconductors. The enhanced adsorptions of the Pt and Au clusters on the $\alpha$-phographene show the probabilities in supporting precious metal species to form high-performance complex catalyst nanostructures, being attractive to call for experimental investigations.

\section{Acknowledgements}

The authors gratefully acknowledge the financial supports from the National Natural Science Foundation of China (NSFC) (Grant No. 11674129 and 11374128).

\section{References}

1 H. W. Kroto, J. R. Heath, S. C. O'Brien, R. F. Curl and R. E. Smalley, Nature, 1985, 318, 162.

2 S. Iijima, Nature, 1991, 354, 56.

3 R. Saito, G. Dresselhaus and M. S. Dresselhaus, Physical Properties of Carbon Nanotubes, Imperial College Press, London, 2003. 
4 K. S. Novoselov, A. K. Geim, S. V. Morozov, D. Jiang, Y. Zhang, S. V. Dubonos, I. V. Grigorieva and A. A. Firsov, Science, 2004, 306, 666.

5 A. T. Balaban, C. C. Rentia and E. Ciupitu, Rev. Roum. Chim., 1968, 13, 231.

6 D. Li, F. Tian, D. Duan, Z. Zhao, Y. Liu, B. Chu, X. Sha, L. Wang, B. Liu and T. Cui, RSC Adv., 2014, 4, 17364.

7 M. J. Xing, B. H. Li, Z. T. Yu and Q. Chen, RSC Adv., 2016, 6, 32740.

$8 \mathrm{X}$. Zhu and M. Wang, RSC Adv., 2016, 6, 112035.

9 Z. G. Fthenakis, RSC Adv., 2016, 6, 78187.

10 H. Lu and S.-D. Li, J. Mater. Chem. C, 2013, 1, 3677.

11 R. H. Baughman, H. Eckhardt and M. Kertesz, J. Chem. Phys., 1987, 87, 6687.

12 N. Narita, S. Nagai, S. Suzuki and K. Nakao, Phys. Rev. B: Condens. Matter Mater. Phys., 1998, 58, 11009.

13 V. R. Coluci, S. F. Braga, S. B. Legoas and D. S. Galvão, Phys. Rev. B: Condens. Matter Mater. Phys., 2003, 68, 035430.

14 A. N. Enyashin and A. L. Ivanovskii, Phys. Status Solidi B, 2011, 248, 1879.

15 Y. Yang and X. Xu, Comput. Mater. Sci., 2012, 61, 83.

16 J. Zhou, K. Lv, Q. Wang, X. S. Chen, Q. Sun and P. Jena, J. Chem. Phys., 2011, 134, 174701.

17 S. W. Cranford and M. J. Buehler, Carbon, 2011, 49, 4111.

18 D. Malko, C. Neiss, F. Viñes and A. Görling, Phys. Rev. Lett., 2012, 108, 086804.

19 B. G. Kim and H. J. Choi, Phys. Rev. B: Condens. Matter Mater. Phys., 2012, 86, 115435.

20 S. Chopra, RSC Adv., 2016, 6, 89934.

21 G. Li, Y. Li, H. Liu, Y. Guo, Y. Li and D. Zhu, Chem. Commun., 2010, 46, 3256.

22 X. Qian, H. Liu, C. Huang, S. Chen, L. Zhang, Y. Li, J. Wang and Y. Li, Sci. Rep., 2014, 5, 7756.

23 M. Long, L. Tang, D. Wang, Y. Li and Z. Shuai, ACS Nano, 2011, 5, 2593.

24 Q. Song, B. Wang, K. Deng, X. Feng, M. Wagner, J. D. Gale, K. Müllen and L. Zhi, J. Mater. Chem. C, 2013, 1, 38.

25 W. Liu, M.-S. Miao and J.-Y. Liu, RSC Adv., 2015, 5, 70766.

26 M. A. Hudspeth, B. W. Whitman, V. Barone and J. E. Peralta, ACS Nano, 2010, 4, 4565.

27 N. N. Karaush, S. V. Bondarchuk, G. V. Baryshnikov, V. A. Minaeva, W.-H. Sun and B. F. Minaev, RSC Adv., 2016, 6, 49505.

28 E. Konstantinova, S. O. Dantas and P. M. V. B. Barone, Phys. Rev. B: Condens. Matter Mater. Phys., 2006, 74, 035417.

29 S. Zhang, J. Zhou, Q. Wang, X. Chen, Y. Kawazoe and P. Jena, Proc. Natl. Acad. Sci. U. S. A., 2015, 112, 2372.

30 B. R. Sharma, A. Manjanath and A. K. Singh, Sci. Rep., 2014, 4, 7164.

31 B. Mandal, S. Sarkar, A. Pramanik and P. Sarkar, Phys. Chem. Chem. Phys., 2013, 15, 21001.

32 V. H. Crespi, L. X. Benedict, M. L. Cohen and S. G. Louie, Phys. Rev. B: Condens. Matter Mater. Phys., 1996, 53, 13303.

33 M. Deza, P. W. Fowler, M. Shtogrin and K. Vietze, J. Chem. Inf. Comput. Sci., 2000, 40, 1325.
34 H. Terrones, M. Terrones, E. Hernández, N. Grobert, J.-C. Charlier and P. M. Ajayan, Phys. Rev. Lett., 2000, 84, 1716.

35 M. J. Bucknum and E. A. Castro, Solid State Sci., 2008, 10, 1245.

36 H. Y. Zhu, A. T. Balaban, D. J. Klein and T. P. Zivkovic, J. Chem. Phys., 1994, 101, 5281.

37 X. Q. Wang, H. D. Li and J. T. Wang, Phys. Chem. Chem. Phys., 2012, 14, 11107.

38 Y. Liu, G. Wang, Q. Huang, L. Guo and X. Chen, Phys. Rev. Lett., 2012, 108, 225505.

39 J. Nisar, X. Jiang, B. Pathak, J. Zhao, T. W. Kang and R. Ahuja, Nanotechnology, 2012, 23, 385704.

40 X.-L. Sheng, H.-J. Cui, F. Ye, Q.-B. Yan, Q.-R. Zheng and G. Su, J. Appl. Phys., 2012, 112, 074315.

41 G. Long, Y. Zhou, M. Jin, M. Jin, B. Kan, Y. Zhao, A. GrayWeale, D. Jiang, Y. Chen and Q. Zhang, Carbon, 2015, 95, 1033.

42 L.-C. Xu, R.-Z. Wang, M.-S. Miao, X.-L. Wei, Y.-P. Chen, H. Yan, W.-M. Lau, L.-M. Liu and Y.-M. Ma, Nanoscale, 2014, 6, 113.

43 C. Su, H. Jiang and J. Feng, Phys. Rev. B: Condens. Matter Mater. Phys., 2013, 87, 075453.

44 C.-P. Tang and S.-J. Xiong, AIP Adv., 2012, 2, 042147.

45 N. V. R. Nulakani, M. Kamaraj and V. Subramanian, $R S C$ $A d v .$, 2015, 5, 78910.

46 X. Q. Wang, H. D. Li and J. T. Wang, Phys. Chem. Chem. Phys., 2013, 15, 2024.

47 Z. Wang, X.-F. Zhou, X. Zhang, Q. Zhu, H. Dong, M. Zhao and A. R. Oganov, Nano Lett., 2015, 15, 6182.

48 L. F. C. Pereira, B. Mortazavi, M. Makaremi and T. Rabczuk, RSC Adv., 2016, 6, 57773.

49 A. Stone and D. Wales, Chem. Phys. Lett., 1986, 128, 501.

50 F. Banhart, J. Kotakoski and A. V. Krasheninnikov, ACS Nano, 2011, 5, 26.

51 J. Lahiri, Y. Lin, P. Bozkurt, I. I. Oleynik and M. Batzill, Nat. Nanotechnol., 2010, 5, 326.

52 J.-H. Chen, G. Autès, N. Alem, F. Gargiulo, A. Gautam, M. Linck, C. Kisielowski, O. V. Yazyev, S. G. Louie and A. Zettl, Phys. Rev. B: Condens. Matter Mater. Phys., 2014, 89, 121407.

53 G. Kress and J. Furthmüller, Phys. Rev. B: Condens. Matter Mater. Phys., 1996, 54, 11169.

54 G. Kress and D. Joubert, Phys. Rev. B: Condens. Matter Mater. Phys., 1999, 59, 1758.

55 J. P. Perdew, K. Burke and M. Ernzerhof, Phys. Rev. Lett., 1996, 77, 3865.

56 H. J. Monkhorst and J. D. Pack, Phys. Rev. B: Condens. Matter Mater. Phys., 1976, 13, 5188.

57 A. Togo, F. Oba and I. Tanaka, Phys. Rev. B: Condens. Matter Mater. Phys., 2008, 78, 134106.

58 G. J. Martyna, M. L. Klein and M. Tuckerman, J. Chem. Phys., 1992, 97, 2635.

59 A. R. Oganov and C. W. Glass, J. Chem. Phys., 2006, 124, 244704.

60 A. R. Oganov, A. O. Lyakhov and M. Valle, Acc. Chem. Res., 2011, 44, 227. 
61 A. O. Lyakhov, A. R. Oganov, H. T. Stokes and Q. Zhu, Comput. Phys. Commun., 2013, 184, 1172.

62 X.-F. Zhou, X. Dong, A. R. Oganov, Q. Zhu, Y. Tian and H.-T. Wang, Phys. Rev. Lett., 2014, 112, 085502.

63 D. M. Ceperley and B. J. Alder, Phys. Rev. Lett., 1980, 45, 566. 64 J. P. Perdew and Z. Zunger, Phys. Rev. B: Condens. Matter Mater. Phys., 1981, 23, 5048.

65 J. P. Perdew and Y. Wang, Phys. Rev. B: Condens. Matter Mater. Phys., 1992, 45, 13244.

66 J. Tao, J. P. Perdew, V. N. Staroverov and G. E. Scuseria, Phys. Rev. Lett., 2003, 91, 146401.

67 J. P. Perdew, A. Ruzsinszky, G. I. Csonka, L. A. Constantin and J. Sun, Phys. Rev. Lett., 2009, 103, 026403.

68 J. Li, Y. Wei, X. Fan, H. Wang, Y. Song, G. Chen, Y. Liang, V. Wang and Y. Kawazoe, J. Mater. Chem. C, 2016, 4, 9613.

69 J. Li, X. Fan, Y. Wei, J. Liu, J. Guo, X. Li, V. Wang, Y. Liang and G. Chen, J. Mater. Chem. C, 2016, 4, 10866.

70 C. G. Lee, X. D. Wei, J. W. Kysar and J. Hone, Science, 2008, 321, 385.

71 M. Born and H. Huang, Dynamical Theory of Crystal Lattices, Clarendon Press, Oxford, 1998.

72 J. Li, X. Fan, Y. Wei and G. Chen, Sci. Rep., 2016, 6, 31840.

73 J. Heyd, G. E. Scuseria and M. Ernzerhof, J. Chem. Phys., 2003, 118, 8207.

74 J. T. Jiang, S. L. Xiu, M. M. Zheng, T. T. Jia, H. Y. Liu, Y. Zhang and G. Chen, Chem. Phys. Lett., 2014, 613, 74.

75 J. Zhou, Q. Wang, Q. Sun, X. S. Chen, Y. Kawazoe and P. Jena, Nano Lett., 2009, 9, 3867.
76 M. M. Zheng, S. J. Li, Y. Su, G. Chen and Y. Kawazoe, J. Phys. Chem. C, 2013, 117, 25077.

77 M. M. Zheng, T. Q. Ren, G. Chen and Y. Kawazoe, J. Phys. Chem. C, 2014, 118, 7442.

78 Q. Sun, Z. Li, D. J. Searles, Y. Chen, G. Lu and A. Du, J. Am. Chem. Soc., 2013, 135, 8246.

79 N. Cheng, S. Stambula, D. Wang, M. N. Banis, J. Liu, A. Riese, B. Xiao, R. Li, T.-K. Sham, L.-M. Liu, G. A. Botton and X. Sun, Nat. Commun., 2016, 7, 13638.

80 M. Zhou, A. Zhang, Z. Dai, C. Zhang and Y. P. Feng, J. Chem. Phys., 2010, 132, 194704.

81 S. L. Xiu, M. M. Zheng, P. Zhao, Y. Zhang, H. Y. Liu, S. J. Li, G. Chen and Y. Kawazoe, Carbon, 2014, 79, 646.

82 G. Chen, S. J. Li, Y. Su, V. Wang, H. Mizuseki and Y. Kawazoe, J. Phys. Chem. C, 2011, 115, 20168.

83 M. Zhou, A. Zhang, Z. Dai, Y. P. Feng and C. Zhang, J. Phys. Chem. C, 2010, 114, 16541.

84 G. Chen and Y. Kawazoe, Phys. Rev. B: Condens. Matter Mater. Phys., 2006, 73, 125410.

85 S. Bulusu, X. Li, L. S. Wang and X. C. Zeng, Proc. Natl. Acad. Sci. U. S. A., 2006, 103, 8326.

86 G. Chen, Q. Wang, Q. Sun, Y. Kawazoe and P. Jena, J. Chem. Phys., 2010, 132, 194306.

87 I. Fampiou and A. Ramasubramaniam, J. Phys. Chem. C, 2012, 116, 6543.

88 G. Chen, P. Jena and Y. Kawazoe, J. Chem. Phys., 2008, 129, 074305. 